\title{
Wavelet analysis applied to magnetograms: singularity detections related to geomagnetic storms
}

\author{
Odim Mendes Jr. \\ National Institute of Space Research - INPE/CEA/DGE \\ 12245-970 São José dos Campos, São Paulo, Brazil \\ Margarete Oliveira Domingues \\ National Institute of Space Research - INPE/CTE/LAC \\ 12245-970 São José dos Campos, São Paulo, Brazil \\ Aracy Mendes da Costa \\ National Institute of Space Research - INPE/CEA/DGE \\ 12245-970 São José dos Campos, São Paulo, Brazil \\ Alícia L. Clúa de Gonzalez \\ National Institute of Space Research - INPE/CEA/DGE \\ 12245-970 São José dos Campos, São Paulo, Brazil
}

\begin{abstract}
When the interplanetary magnetic field carried by the solar wind opposes to the Earth's intrinsic magnetic field, a substantial transfer of energy into the terrestrial magnetosphere takes place. If this condition persists for several hours, the magnetosphere becomes very disturbed. As a result at mid-to low latitudes a ring current starts to develop and at high latitudes ionospheric currents (electrojet and field-aligned currents) dominate. The ring current provides the geomagnetic conditions for magnetic storms to be settled down. Wavelet analysis is becoming a common tool since they allow the decomposition of data, functions or operators into different frequency or scale components. Accordingly, wavelet transforms seem to be good tools to "zoom in" short-lived high frequency phenomena such as discontinuities ("shocks") in signals and transient structures. In this work the remarkable ability of wavelets to highlight the singularities associated with discontinuities present in the horizontal component of the Earth magnetic field is explored. Magnetograms obtained at five magnetic stations for two geomagnetic storms have been analyzed by a Daubechies orthogonal wavelet transform decomposition. The wavelet coefficient magnitudes at three levels have been studied. In both cases, the physical discontinuities in the horizontal component of the geomagnetic field are clearly detected by means of these coefficients identifying the disturbed interval related to geomagnetic storms. Wavelet analysis has proved to be a useful tool in the identification of the geomagnetic storms just using non-processed data.
\end{abstract}


Key words: wavelet analysis, multi-resolution analysis, geomagnetic storm, sun-Earth coupling, planetary electrodynamics

\section{Introduction}

Under suitable geometric and energetic conditions, a disturbed solar wind plasma flowing out from the Sun can reach the Earth environment. When the interplanetary magnetic field (IMF) carried by the solar wind opposes the Earth's intrinsic magnetic field, a substantial transfer of energy into the terrestrial magnetosphere occurs. If this condition persists for several hours, the magnetosphere becomes very disturbed (Gonzalez et al. 1994, Cluá de Gonzalez et al. 2004). In consequence of an enhanced level of solar wind-magnetosphere coupling, normally existing magnetospheric and ionospheric quiet currents are widened and intensified (Jankovicová et al. 2002). The ring current dominates at middle and low latitudes and a system of ionospheric electrojet currents flowing horizontally in the auroral oval dominates at higher latitudes. These currents systems characterize situations called respectively geomagnetic storms and geomagnetic substorms. Another current system provides a link between the high and the low latitudes current systems: the field-aligned currents, which contributes to increase the complexity of these current systems. The degree of relative contribution of the ionospheric current system against the magnetospheric current system in magnetic observations on the ground magnetic observations may be an important feature that can be examined through the characteristics of the storm sudden commencement (SSC) (Tsunomura 1998). All these currents affect especially the horizontal component of the geomagnetic field at the Earth's surface. This component is used in the calculation of the Dst index, that describes the variations in in the equatorial ring current (Sugiura \& Kamei 1991), and the $A E$ index, that describes the disturbances in the auroral electrojet current system (Mayaud 1980).

When hot ions are injected into the inner magnetosphere, the geometry of the geomagnetic field causes them to drift around the Earth forming a westward ring current ( $\mathrm{O}^{\prime}$ Brien \& McPherron 2000). This current is composed by linear and nonlinear processes from the dawn-dusk electric field. Although the injection of particles and its magnetic effect may be approximated as a linear process, the trapping mechanism and hence the resultant ring current intensity may not be approximated as a linear process since it depends on the history of the cross tail potential difference. This effect will limit the efficiency of the linear prediction filter of the ring current from the solar wind parameters (Takahashi et al. 1990). Forecasting the state of the ring current is a necessity to forecast the magnetic field in the magnetosphere. The Dst index represents the longest commonly used measure of the state of the ring current and therefore it is essential in such forecasting (Baker 1998). The magnetic field variation produced by the ring current decreases the magnetic field at 
the Earth's surface and this depression is measured by the Dst index (Sugiura \& Kamei 1991).

The magnetic field measured at mid latitudes can be affected significantly by variations of the solar wind ram pressure, which produces changes in the magnetopause current.current. This process gives place to an SSC, when an increase in the horizontal magnetic field is observed at middle-to-low latitudes. It is generally considered that the solar wind ram pressure represents only a linear correction to the magnetometer measurements. This contribution can be dynamical in nature and spatially dependent, since the compression of the magnetopause can affect the magnetometer locations with different strength (Valdivia et al. 1999).

A typical storm includes a substantial ring current that develops over a few hours and then recovers over several days (Kamide et al. 1997). Geomagnetic disturbances observed at mid-to-low latitudes show less spatial irregularities than those observed at high latitudes, because theses regions are far away from the origins of disturbance and they are not directly linked to the interplanetary magnetic field. The magnetic variations at low latitude, ordinarily used to derive the Dst index, are regarded as representing the whole magnetospheric process without severe locality. However, the magnetic disturbances at middle and low latitudes exhibit a rather complicated time and/or spatial variation.

Wavelet analysis is becoming a common tool since it allows the decomposition of data, functions, or operators into different frequency or scale components (Strang \& Nguyen 1996, Foufoula-Georgiou \& Kumar 1995, Daubechies 1992, Ruskai \& et al. 1992, Chui 1992a,b). Each component can then be studied with a resolution that matches its scales, i.e. at high frequency the wavelet is very narrow, while at low frequency the wavelet is broad. As a result, wavelet transforms are good tools to "zoom in" on short-lived high-frequency phenomena, such as singularities in signal and transient structures. As each scale of the wavelet transform is associate with a frequency-band, the wavelet transform decompositions represent well the frequencies detected in the geomagnetic storms. In a particular scale, because of the wavelet analysis properties, the wavelet coefficients will have small amplitudes (Meyer 1990), where the magnetic field is 'smooth'. On the other hand they will have larger amplitudes where singularities and transient structures occur in the magnetograms. Some applications to geomagnetic field data have been reported by Bayer et al. (2001) and in the references therein.

In order to detect the variations of the horizontal component of the geomagnetic field related to geomagnetic storms, singularities have been analyzed by means of wavelet technique. The purpose of this work is to present an alternative way to identify quiescent and non-quiescent periods relate to geomagnetic storms using magnetograms instead of the processed Dst index. 


\section{Wavelet Technique Applied to Singularity Detections}

The wavelet transform is a linear transform with the property of being covariant under translation and dilatation that can be used in the analysis of non-stationary signals to obtain information on the frequency variations of these signals and to detect their structures localization in time and/or in space. The time/space localization occurs because the wavelet function is defined in a finite interval. In a general way, wavelets have both time-frequency localization, with time resolution inversely proportional to frequency resolution

$$
\Delta^{j} t \times \Delta^{j} \xi=\text { constant }
$$

as illustrated by Figure 1. The left panel of this figure shows a diagram of the time-frequency plane $(t \times \xi$ plane). In the right panel a zoom out scheme of a wavelet function dilatation in this plane is shown for three different j-levels. It can be seen from this time-frequency plane that as the levels (scale) increase, the time resolution decreases.

In wavelet analysis, signals $f(t)$ are represented by series like

$$
f(t)=\sum_{j=-\infty}^{\infty} \sum_{k=-\infty}^{\infty} d_{k}^{j} \psi_{k}^{j}(t)
$$

where $\psi_{k}^{j}(t)=\psi\left(2^{j} t-k\right)$ are called mother wavelet (Daubechies 1992, Chui $1992 b)$. The wavelet coefficients $d_{k}^{j}$ are expressed by

$$
d_{k}^{j}=2^{j} \int_{-\infty}^{\infty} f(t) \psi\left(2^{\ell} t-k\right) d t .
$$

In a multi-level basis, wavelet coefficients are also known as "details" because they can be seen as the difference between the signal in two consecutive scale levels. Actually, the wavelet transform is a map of the signal to its wavelets coefficients. It could be proved that the amplitudes of the wavelet coefficients are directly proportional to the local smoothness of the function it represents (Meyer 1990).

The wavelet transform is equivalent to a mathematician microscope, which a magnification given by the inverse of the dilatation parameter and the optical ability, by the choice of the mother-wavelet function (Foufola-Georgoiu \& Kumar 1994).

It is possible to build up wavelet functions using a mathematical tool known as multi-resolution analysis formed by a pair $\left\{V^{j}, \phi^{j}\right\}$, in such a way that there are sequences of embedded approximating spaces $V^{j} \in V^{j+1}$ and the functions $\phi_{k}^{j}$ form a Riesz basis for $V^{j}$ of $L^{2}(\mathbf{R} / \mathbf{Z})$ (Mallat 1991, Daubechies 1992, Jawerth \& Sweldens 1994). In such formalism,

$$
V_{j}=\operatorname{span}\left\{\phi_{k}^{j}(x)\right\} .
$$


In this technique, a mother-wavelet function is generated from a scaling function that obeys the scale relation

$$
\phi(x)=2 \sum_{k} h(k) \phi(2 x-k),
$$

where $\phi(x)$ is known as the scale function, and $h(k)$ is a low pass filter. Then, the mother-wavelet functions are built as

$$
\psi(x)=\sum_{k} g(k) \phi(2 x-k),
$$

where $g(k)=(-1)^{k+1} h(1-k)$ is a high-pass band filter. From this motherwavelet, it is possible to build up functions $\psi_{k}^{j}$ that can be dilated and contracted. They also form a Riesz basis for the "detail" spaces $W^{j}=V^{j+1}-V^{j}$. This represents the difference of information between $V^{j}$ and $V^{j+1}$, i.e.,

$$
\left(\Pi^{j+1}-\Pi^{j}\right) f(x)=Q^{j} f(x),
$$

where the projections are

$$
\Pi^{j} f(x)=\sum_{k}\left\langle f, \phi_{k}^{j}\right\rangle \phi_{k}^{j}(x)
$$

and the details are

$$
Q^{j} f(x)=\sum_{k}\left\langle f, \psi_{k}^{j}\right\rangle \psi_{k}^{j}(x)
$$

The Daubechies orthogonal wavelet functions are examples of this type of construction. They form an orthogonal system, i.e.,

$$
\int_{-\infty}^{\infty} \psi\left(2^{j} x-k\right) \psi\left(2^{\ell} x-n\right) d x=\left\{\begin{array}{cl}
2^{-j} & \text { if } j=\ell \text { and } k=n \\
0 & \text { otherwise }
\end{array}\right.
$$

This means that no redundant information is stored. Those functions do not have analytical expressions and are not symmetric. The $h(k)$ coefficients are zero for $k<0$ and for $k \geq 2 K$, where $K$ is related to the smoothness order of the wavelet. The support of $\phi=\phi_{K}$ is $\left[0, \frac{K}{2}-1\right]$. For $K=2$,

$$
\begin{gathered}
h(0)=0.341506350946110, \quad h(1)=0.591506350946109, \\
h(2)=0.158493649053890, \quad h(3)=-0.0915063509461096 .
\end{gathered}
$$

For the purposes of this work, the Daubechies orthogonal wavelet with $K=2$ was chosen because:

a) this wavelet family is slightly asymmetric, thus a "future time/space" information is used to calculate the wavelet coefficients; 
b) this wavelet family is adequated to detect shock-like singularities, because it uses few coefficients and it is a good representation of low order polynomials.

The methodology for processing the data sets was based on the following steps:

1) The largest amplitudes of the wavelet coefficients $d_{k}^{j}$ are analyzed in the three first decomposition levels, a cut-off threshold is chosen for each level. In a general way the threshold is defined as the minimum value of the coefficient above the baseline.

2) The wavelet coefficient are restored leading to zero the coefficients less or equal to the chosen threshold and then an inverse wavelet transform is processed. The original and the reconstructed time series are compared to verify the amount of the energy lost in the reconstructed series.

3) If a little bit of energy is lost, the position of the largest amplitude-remained wavelet coefficients after the threshold cut-off process indicate the "shock" candidate regions.

The essential feature of wavelets used in this work is their ability to highlight the singularities associated with "shocks" (discontinuity) present in the horizontal component of the Earth's magnetic field. In order to apply the wavelet singularity detection technique to geomagnetic field recorded by magnetograms, time intervals associated with geomagnetic storms were selected for tests.

\section{Data sets}

To illustrate the singularities detection related to geomagnetic storms using the wavelet analysis, two geomagnetic storms were selected. These events occurred near solar maximum period (solar cycle 21). The selected time intervals included the geomagnetic disturbance and a previous period of relatively low geomagnetic activity. The first geomagnetic storm, discussed in details by Mendes (1992), started on November 7, 1978 at $22: 27 \mathrm{UT}$ and corresponds to a moderate magnetic storm with a maximum $D s t=-47 \mathrm{nT}$ at $02: 00 \mathrm{UT}$ on November 8 . The second one started on August 29, 1979 with a minimum $D s t=-140 \mathrm{nT}$ at $19: 00 \mathrm{UT}$ and corresponds to an intense magnetic storm, discussed in details by (Tsurutani et al. 1988).

In order to develop this analysis, one minute time resolution magnetograms obtained at five magnetic stations were used. A six-day interval of the geomagnetic field components $\mathrm{H}$ or $\mathrm{X}$, for low and high latitudes respectively, was considered as the dataset. The geographic and geomagnetic coordinates and magnetic local time $(\mathrm{MLT})^{1}$ of these magnetic stations are given in Table 1 . The magnetic stations

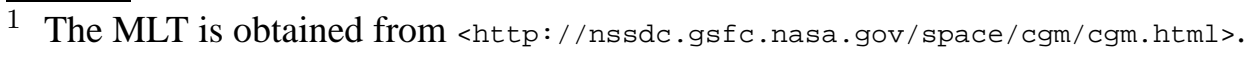


Kakioka, Hermanus and Boulder are located at medium and low latitudes and are longitudinally worldwide uniformly spaced. The auroral stations Fort Churchil and Dumont d'Urville located in the north and south hemispheres, respectively, were included for comparison purposes. It is important to mention that in the derivation of the standard Dst index, in the 1978-1979 period, only four magnetic stations were used: Honolulu, Kakioka, Hermanus and San Juan (Iyemori et al. 1998). Boulder was included in this study, because the data from Honolulu and San Juan were not available at WDC (2002), for the selected periods.

Figure 2 shows the magnetograms obtained at Fort Churchill, Boulder, Kakioka, Hermanus and Dumont d'Urville together with $A E$ and Dst indices, for the moderate and intense geomagnetic storms respectively in (a) and (b). In both cases the geomagnetic storm (magnetically disturbed period) is preceded by a quiet period as seen by $D s t$ index. The $A E$ index also represents the geomagnetic activity, although restricted to the the auroral regions. As it can be noticed from this figure, geomagnetic disturbances are recorded by the stations in a non-identical display, although a common behavior could be identified. The signatures presented by the magnetograms are not similar even for the medium and low latitude stations.

\section{Results and discussion}

In Figure 2, the signatures recorded by each magnetic station are quite dissimilar. One question arises when analyzing these non-processed data: Is there any common features among these magnetograms related to geomagnetic storms? Can any resources offered by the wavelet analysis be explored to elucidate this issue? The idea is to use the wavelet technique to unveil some common features of these signatures. The signals were then submitted to the wavelet analysis and the singularities detected were compared in order to identify any peculiar feature.

Figure 3 shows the behavior of the wavelet coefficient for the moderate storm of November, $7-8,1978$. In each panel, from top to bottom, are shown, the Dst index, the $\mathrm{X}$ or H-component of the geomagnetic field and the first three levels of the wavelet coefficients of the discrete wavelet transform (major levels considered for the purpose of this work). The letters (a) to (e) stand for stations Fort Churchill, Boulder, Kakioka, Hermanus and Dumont d'Urville respectively. Taking into account that the highest amplitudes of the wavelet coefficients indicate singularities, in all cases of the Figure 3 singularity structures were found in association with the geomagnetic storm. When the magnetosphere is under quiet conditions (magneticaly quiescent periods) the horizontal components of the geomagnetic field recorded in the magnetograms can be represented by smooth functions. In such cases the wavelet coefficients show very small values. On the other hand, when a geomagnetic disturbance is under development (disturbed periods) the wavelet coefficients are significantly bigger. These coefficients are able to identify the sud- 
den variations that occur in the geomagnetic field components. In this work, the wavelet coefficient amplitudes will not be investigated, since its purpose is only to characterize the quiescent and non-quiescent periods.

This result can be used as a marker of the geomagnetic activity, an indicator that some process of energy transfer is going on. A straightforward result of the wavelet technique applied to the dataset is that it allows to distinguish quiescent from nonquiescent periods. The wavelet coefficients of the first decomposition levels of the wavelet transform shows indeed a better time localization and then are locally associated with higher frequencies of the geomagnetic disturbance. In the two cases studied, cases the first three decomposition levels have proved to be sufficient to isolate the singularity structures. One can observe that the magnetic stations do not show the same singularity configuration in different levels. This behavior may be related to differences in the magnetic coordinates, type of magnetometer used, local time, ground conductivity and Sq current effects.

Figure 4 is similar to Figure 3, but refers to the intense geomagnetic storm of 29-30 August, 1979. Also in this case a singularity structure could be observed for all the magnetograms considered.

As shown by Figure 3 and Figure 4 , it was possible to identify singularity structures related to magnetic storm time intervals in the magnetograms of the magnetic stations considered. The highest relative amplitudes of the coefficients are coincident in time. Accordingly, the structures allow the identification of quiescent and non-quiescent periods in the horizontal component of the geomagnetic field signals independently on the general signature of the magnetic station considered. Indeed, using this tool, the intrinsic processes of energy transfer are being surveyed.

From the present analysis it seems that for higher latitude magnetic stations (FCC, BOU and DRV), larger amplitude wavelet coefficients are more frequent in the first two decomposition levels, while for lower stations (KAK and HER) the most significant decomposition levels for the geomagnetic storm identification seem to be the second and the third.

This fact, actually, confirms the already known concepts that at higher latitudes the penetration of charged particles and the energy injection are characterized by phenomena that involve high-frequency signals; while at lower latitudes coupling processes do exist that attenuate high-frequency signals (Morioka et al. 2003).

Magnetograms obtained at DRV show more transient variations in the decomposition levels of the wavelet transform in the considered time intervals than those obtained at FCC. This suggests an ivestigation on the symmetry level of the energetic source in relation to these magnetic stations. Seasonal effects can also play a significant role in the observed signal display. In relation to the lower latitude stations used in the Dst calculation, it was noticed that in the three stations used in this analysis (HER, BOU and KAK) the singularities are more clearly seen during 
the main phase of the geomagnetic storm. It is also worthwhile to mention that in the multi-scale analysis of the $A E$ index (not shown in this work), this effect has also been detected and other singularities were found in that signal. In these cases, a poorer temporal localization of the structures was noticed in the analyzed signal. The same effect was observed at FCC and DRV and more transients were observed in the latter station. This may confirm that an injection process in the ring current is associated to an injection process in the auroral region, but the opposite may not be true (Tsurutani et al. 1997).

\section{Conclusions}

The Daubechies orthogonal wavelet analysis allowed to localize structures with the highest wavelet coefficients and to remark that they were coincident with the geomagnetic storm periods. The first three decomposition levels, related to the magnetograms obtained at different locations, are sufficient to identify the common singularities among the records at a given period. This facilitates the visualization of shocks (singularities) in this type of data. Based on its ability to provide an objective analysis, this wavelet technique has revealed a helpful tool in the study of magnetospheric phenomena such as the time localization of geomagnetic storms. As subsidiary result, it was observed that the non-quiescent period is related to the main phase of geomagnetic storms.

From the comparison between high to low latitude stations, it is also worthwhile to mention that, in the multi-scale analysis of the magnetometer data, some features have been detected. In both, the maxima amplitude of the wavelet coefficients are localized in the main part of the geomagnetic storms. On the other hand, in the high latitudes, other high values of the wavelet coefficients appear. The behavior associated with higher latitude stations (FCC e DRV) and also by the AE index suggests that the energy transfer processes are not symmetric. This may confirm that an injection process in the ring current is associated with an injection process in the auroral region, but the opposite may not be true.

The highest amplitude wavelet coefficients allow to define a threshold that helps to identify the the time localization of the singularities associated with the geomagnetic storms. A further analysis of this threshold is being developed by the authors. Once the threshold is settle down it will be easy to isolate the most relevant singularities from those less relevant and probably identify the perturbation regimes.

\section{References}

Baker, D. N. (1998), 'What is space weather?', Advances in Space Research 22, 7 16. 
Bayer, M., W., F. \& Maier, T. (2001), 'A vector wavelet approach to iono- and magnetospheric geomagnetic satellite data', Journal of Atmospheric and SolarTerrestrial Physics.

Chui, C. (1992a), An Introdution to Wavelets, Vol. 1, Academic Press.

Chui, C., ed. (1992b), Wavelets:A tutorial in theory and applications, Vol. 2, Academic Press.

Cluá de Gonzalez, A. L., Mendes da Costa, A. \& Gonzales, W. D. (2004), 'Ring current space-time inhomogeneities in intense geomagnetic storms', Geofísica Internacional 43(2), 205-215.

Daubechies, I. (1992), Ten Lectures on Wavelets, Vol. 61 of CBMS-NSF Regional Conference (Series in Applied Mathematics), SIAM, Philadelphia, PA.

Foufola-Georgoiu, E. \& Kumar, P., eds (1994), Wavelets in Geophysics, Vol. 4 of Wavelet Analisys and its Applications, Academic Press.

Foufoula-Georgiou, E. K. \& Kumar, P., eds (1995), Wavelet in Geophysics, Academic Press. 373 pp.

Gonzalez, W. D., Joselyn, J. A., Y., K., Kroehl, H. W., Rostoker, G., Tsurutani, B. T. \& Vasyliunas, V. M. (1994), 'What is a geomagnetic storm?', Journal Geophysics Research 99(A4), 5771-5792.

Iyemori, T., Araki, T., Kamei, T. \& Takeda, M. (1998), 'Mid-latitude geomagnetic indices asy and sym 1997', Data Analysis Center for Geomagnetism and Space Magnetism, Kyoto University, Japan. (Number 8).

Jankovicová, D., Dolinský, P., Valach, F. \& Vörös, Z. (2002), 'Neural networkbased nonlinear prediction of magnetic storms', Journal of Atmospheric and Solar-Terrestrial Physics.

Jawerth, B. \& Sweldens, W. (1994), 'An overview of wavelet based multiresolution analyses', SIAM Review 36(3), 377-412.

Kamide, Y., Baumjohann, W., Daglis, I. A., Gonzalez, W. D., Grande, M., Joselyn, J. A., McPherron, R. L., Phillips, J. L., Reeves, E. G. D., Rostoker, G., Sharma, A. S.and Singer, H. J., Tsurutani, B. \& Vasyliunas, V. M. (1997), 'Current understanding of magnetic storms:storm/substorm relationships', Journal Geophysics Research.

Mallat, S. (1991), 'Multiresolution approximations and wavelets orthonormal bases', Transaction of American Mathematical Society 315, 334-351.

Mayaud, P. N. (1980), Derivation, meaning, and use of geomagnetic indices, Vol. 22 of Geophysical Monograph, American Geophysical Union, Washington, DC.

Mendes, O. J. (1992), A origem interplanetária e o desenvolvimento da fase principal das tempestades geomagnéticas moderadas (1978-1979), PhD thesis, INPE, São José dos Campos. INPE-5445-TDI/491.

Meyer, Y. (1990), Ondelettes et Operateurs, Hermann, Paris.

Morioka, A., Miyoshi, Y., Seki, T., Tsuchiya, F., Misawa, H., Oya, H., Matsumoto, H., Hashimoto, K., Mukai, T., Yumoto, K. \& Nagatsuma, T. (2003), 'AKR disappearance during magnetic storms', Journal of Geophysical Research 108(A6), 1226-1235.

O’Brien, T. \& McPherron, R. L. (2000), 'Forecasting the ring current index dst in 
real time', Journal of Atmospheric and Solar-Terrestrial Physics 62, 1295-1299.

Ruskai, M. B. \& et al., eds (1992), Wavelets and their applications, Jones and Bartlett, Boston, Ma. (515'.2433-dc20).

Strang, G. \& Nguyen, T. (1996), Wavelet and filters bank, Wellesley-Cambridge, Cambridge.

Sugiura, M. \& Kamei, T. (1991), 'Equatorial dst index 1957-1986', ISGI Publications Office.

Takahashi, S., Iyemori, T. \& Takeda, M. (1990), 'Ring current response to impulsive southward imf: A cause of second development of the dst index', Journal Geomagnetism Geoelectricity 42, 1325-1331.

Tsunomura, S. (1998), 'Characteristics of gepmagnetic sudden commencement observed in middle and low latitudes', Earth Planets Space 50, 755-772.

Tsurutani, B. T., Gonzalez, W. D., Kamide, Y. \& Arballo, J. K., eds (1997), Magnetic Storms, Geophysical Monograph 98, American Geophysical Union, Washington, chapter The role of substorms in generation of magnetic storms.

Tsurutani, B. T., Gonzalez, W. D., Tang, F., Akasofu, S. I. \& Smith, E. J. (1988), 'Origin of interplanetary southward megnetic fields responsible for major magnetic storms near solar maximum (1978-1979)', Journal Geophysics Research.

Valdivia, J. A., Vassiliadis, D. \& Klimas, A. (1999), 'Spatiotemporal activity of magnetic storms', Journal Geophysics Research 104(6), 12239-12250.

WDC (2002). <http://swdcwww.kugi.kyoto-u.ac.jp/index.html>.

\section{Acknowledgements}

\section{List of Tables}

1 Magnetic Stations Considered in the Analysis

\section{List of Figures}

1 The left panel shows the time-frequency plane $(t \times \xi$ plane $)$ and the right panel shows a zoom-out scheme of wavelet function dilatation in this plane for three different j-levels.

2 DST and AE indices and horizontal component magnetograms obtained at DRV, HER, KAK, BOU and FCC for (a) Event I and (b) Event II. 
3 Geomagnetic field data set for November, $4-9,1978$. The letters (a) to (e) stand for stations Fort Churchill, Boulder, Kakioka, Hermanus and Dumont d'Urville. Each panel shows from top to bottom, the Dst index, the $\mathrm{X}$ or $\mathrm{H}$-component of the geomagnetic field and the first three levels of the wavelet coefficients of the discrete wavelet transform.

4 Geomagnetic field data set for August, $26-31,1978$. The letters (a) to (e) stand for stations Fort Churchill, Boulder, Kakioka, Hermanus and Dumont d'Urville. Each panel shows from top to bottom, the Dst index, the $\mathrm{X}$ or $\mathrm{H}$-component of the geomagnetic field and the first three levels of the wavelet coefficients of the discrete wavelet transform. 

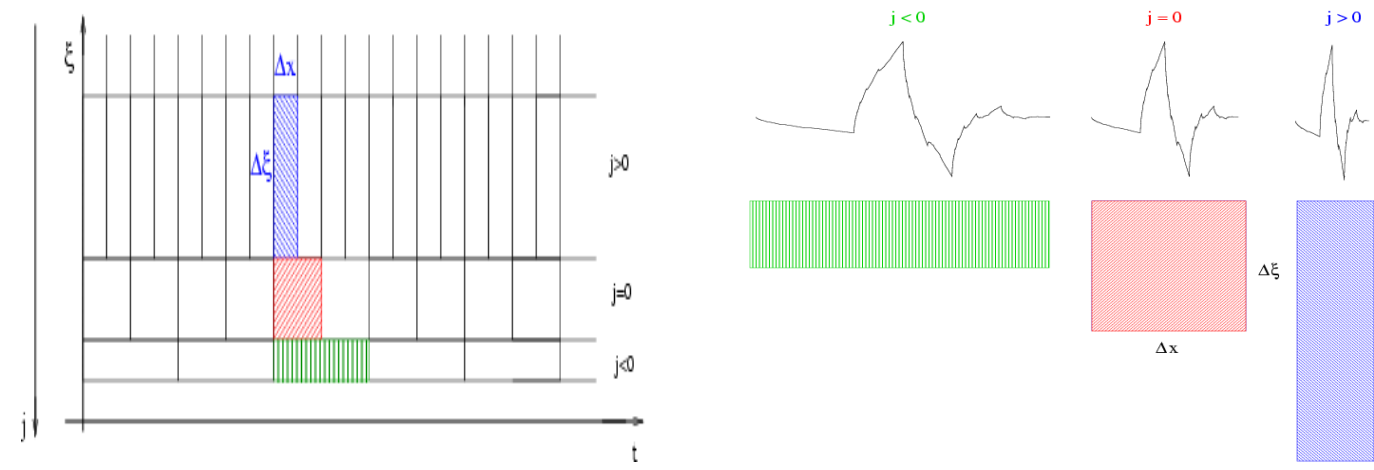

Fig. 1. The left panel shows the time-frequency plane $(t \times \xi$ plane $)$ and the right panel shows a zoom-out scheme of wavelet function dilatation in this plane for three different $j$-levels.

Table 1

Magnetic STATIOnS CONSIDERED IN THE ANALYSiS

\begin{tabular}{l|rr|rr|r}
\hline \hline \multirow{2}{*}{ Station } & \multicolumn{2}{|c|}{ Geog. Coord. } & \multicolumn{2}{|c|}{ Geom. Coord., (1979) } & MLT \\
\cline { 2 - 6 } & Lat. $\left(^{o}\right)$ & Long. $\left({ }^{\circ}\right)$ & Lat. $\left({ }^{o}\right)$ & Long. $\left(^{o}\right)$ & (when UT=0) \\
\hline Ft Churchil (FCC) & 58.80 & 265.90 & 69.83 & 329.12 & $6: 40$ \\
Boulder (BOU) & 40.13 & 254.77 & 49.32 & 317.75 & $7: 26$ \\
Kakioka (KAK) & 36.23 & 140.18 & 28.78 & 210.69 & $15: 06$ \\
Hermanus (HER) & -34.42 & 19.23 & -42.02 & 80.84 & $23: 48$ \\
Dumont d'Urville (DRV) & -66.66 & 140.01 & -80.60 & 235.10 & $12: 53$ \\
\hline \hline
\end{tabular}


(a) 4-9 Nov, 1978

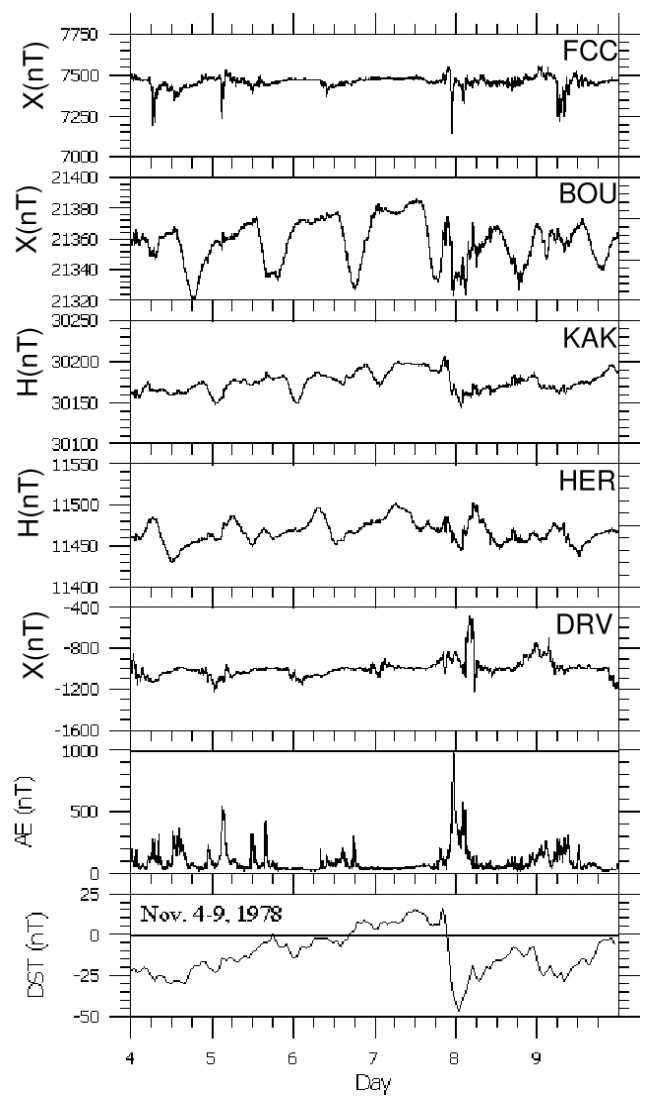

(b) 26-31 Aug, 1979

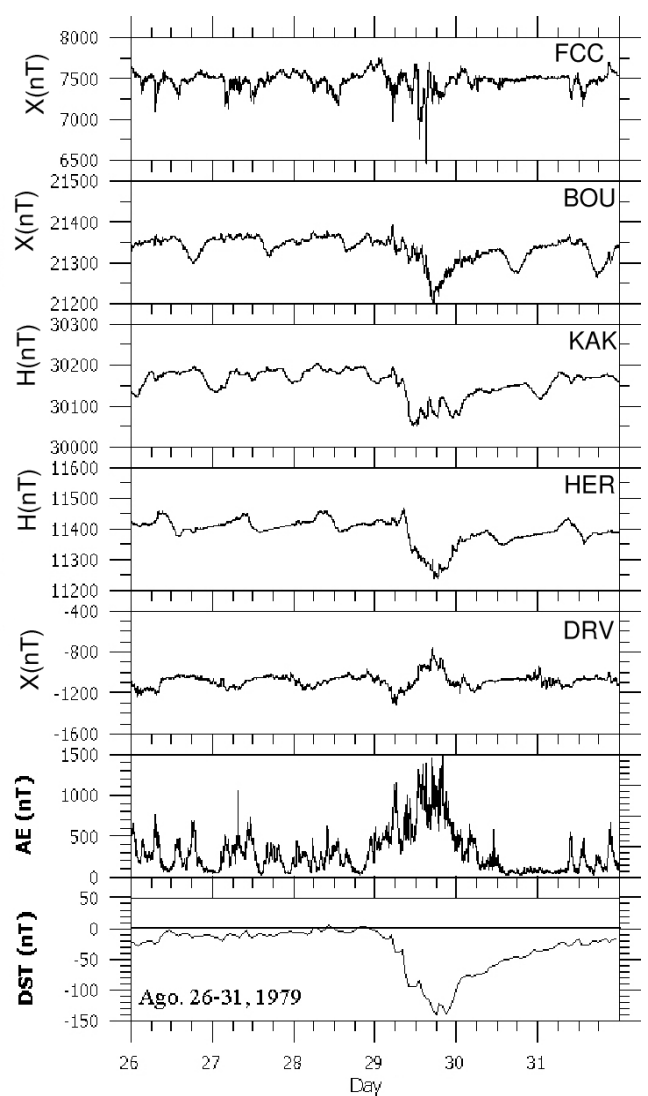

Fig. 2. DST and AE indices and horizontal component magnetograms obtained at DRV, HER, KAK, BOU and FCC for (a) Event I and (b) Event II. 
(a) FCC

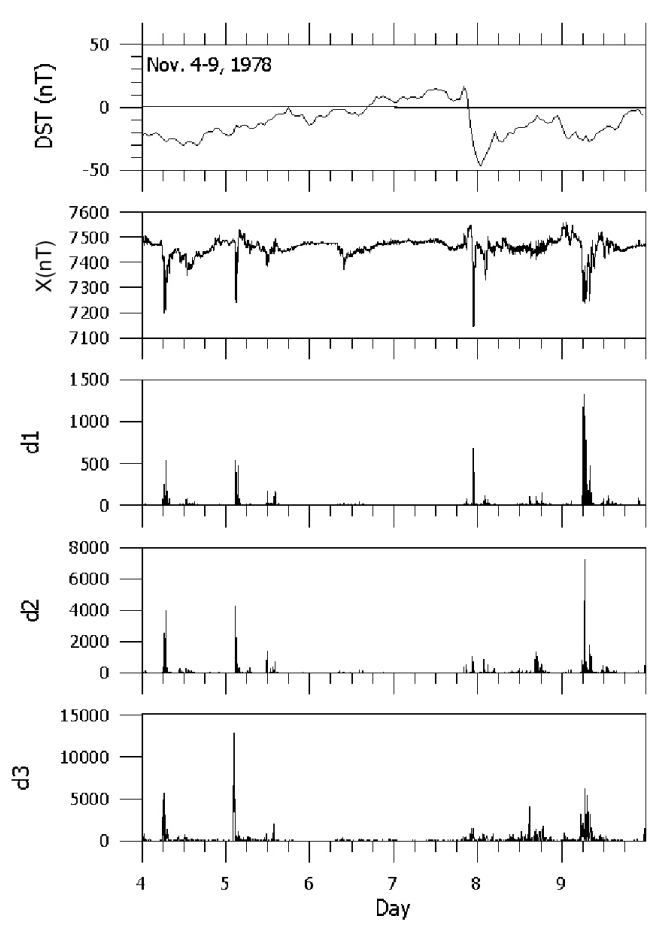

(c) KAK

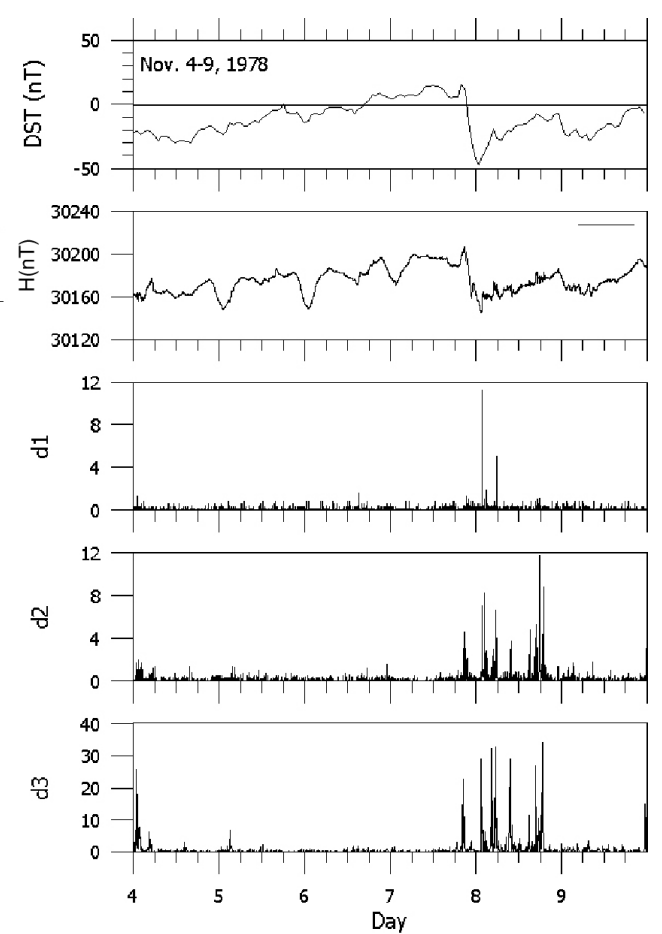

(b) $\mathrm{BOU}$

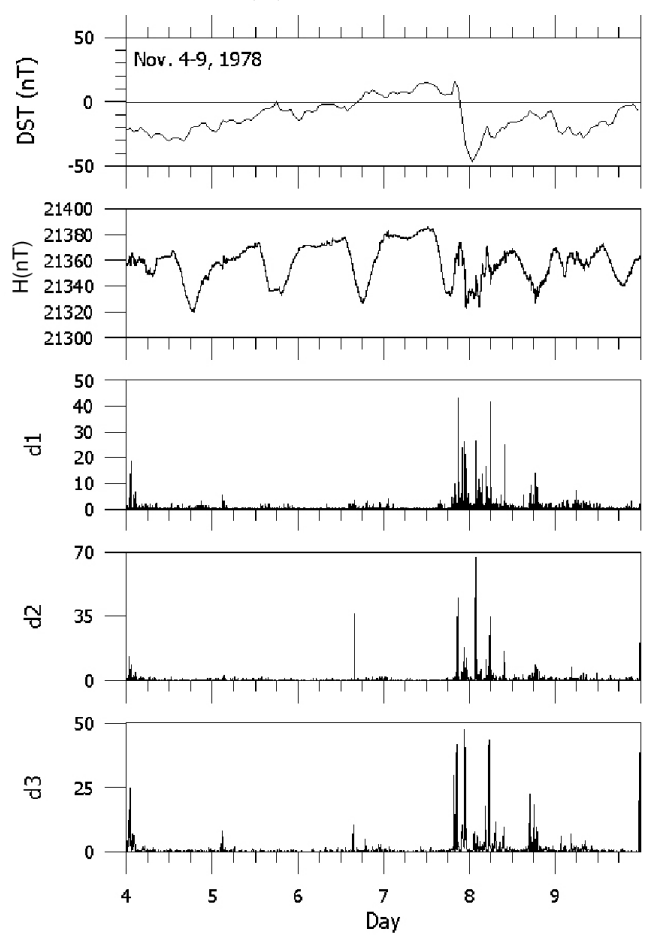

(d) HER

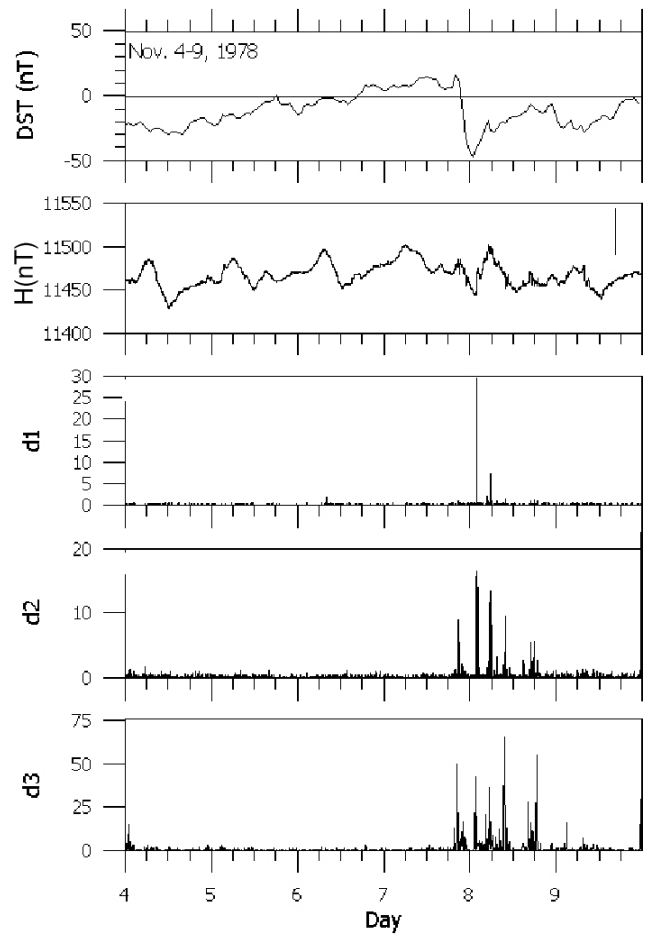


(e) DRV

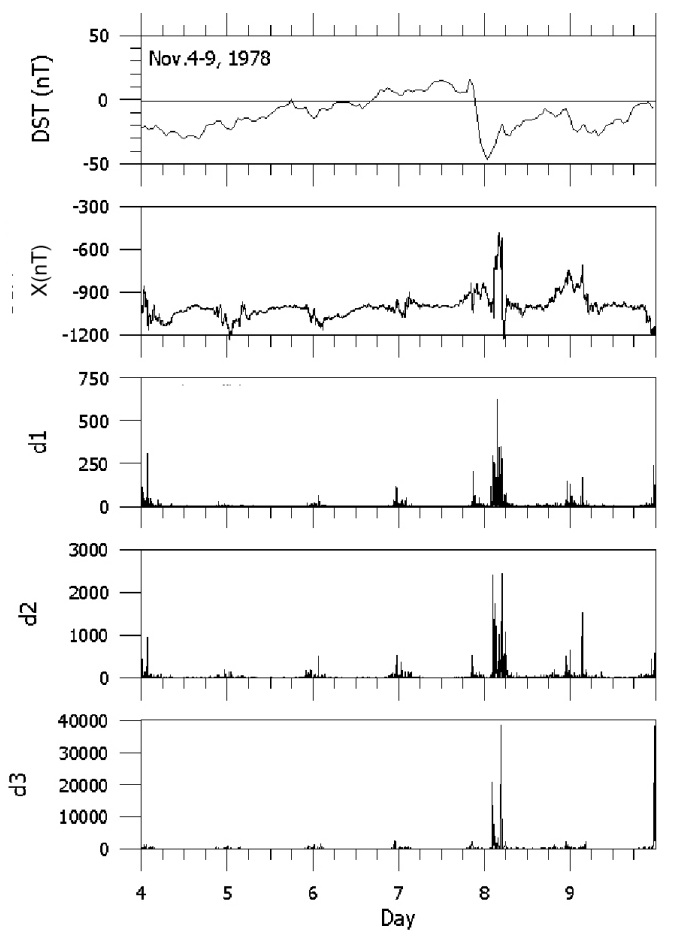

Fig. 3. Geomagnetic field data set for November, 4-9, 1978. The letters (a) to (e) stand for stations Fort Churchill, Boulder, Kakioka, Hermanus and Dumont d'Urville. Each panel shows from top to bottom, the Dst index, the $\mathrm{X}$ or $\mathrm{H}$-component of the geomagnetic field and the first three levels of the wavelet coefficients of the discrete wavelet transform. 
(a) FCC

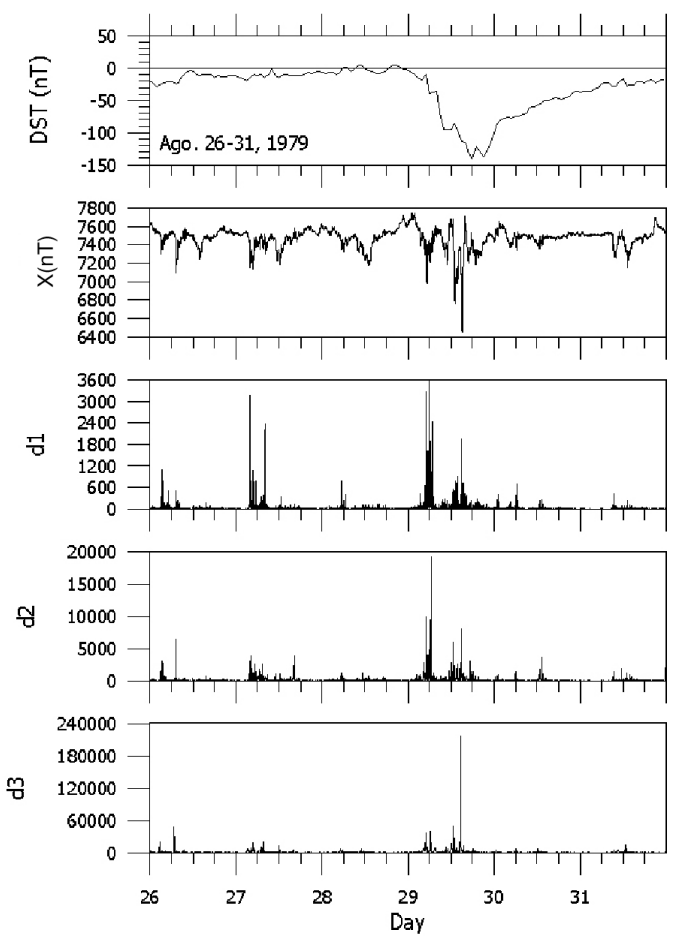

(c) KAK

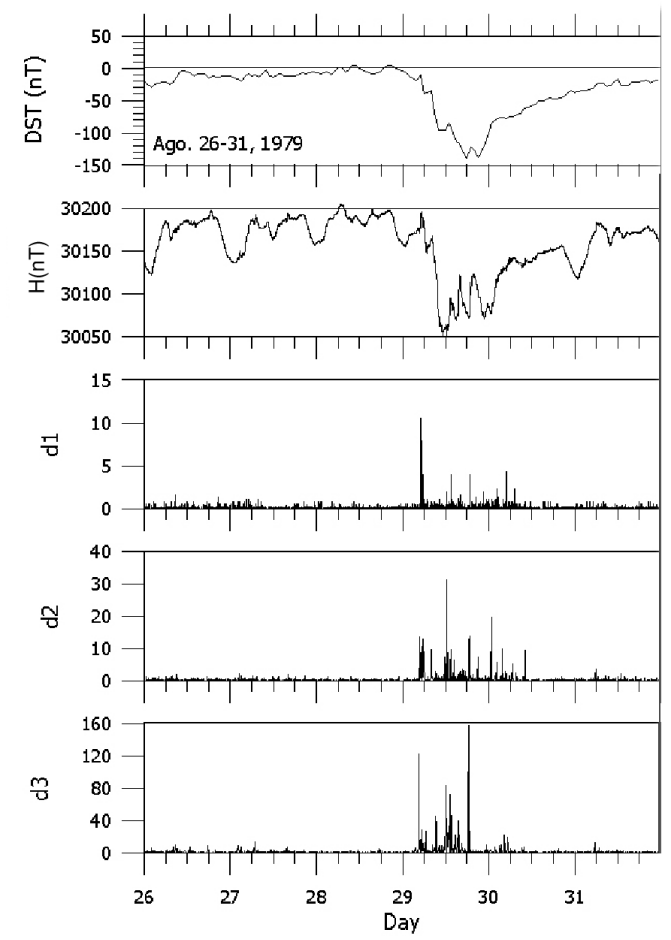

(b) $\mathrm{BOU}$

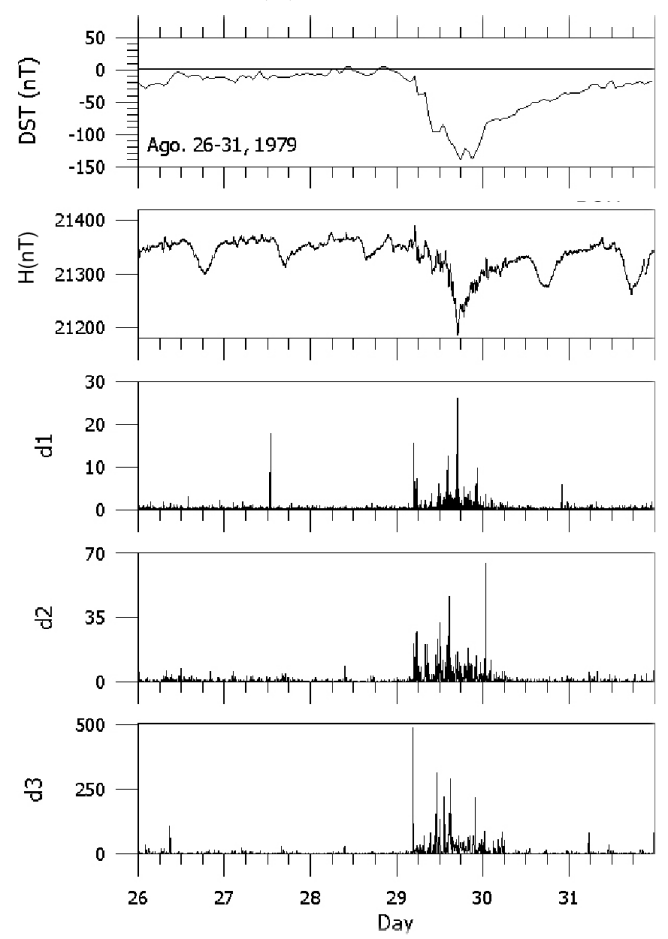

(d) HER

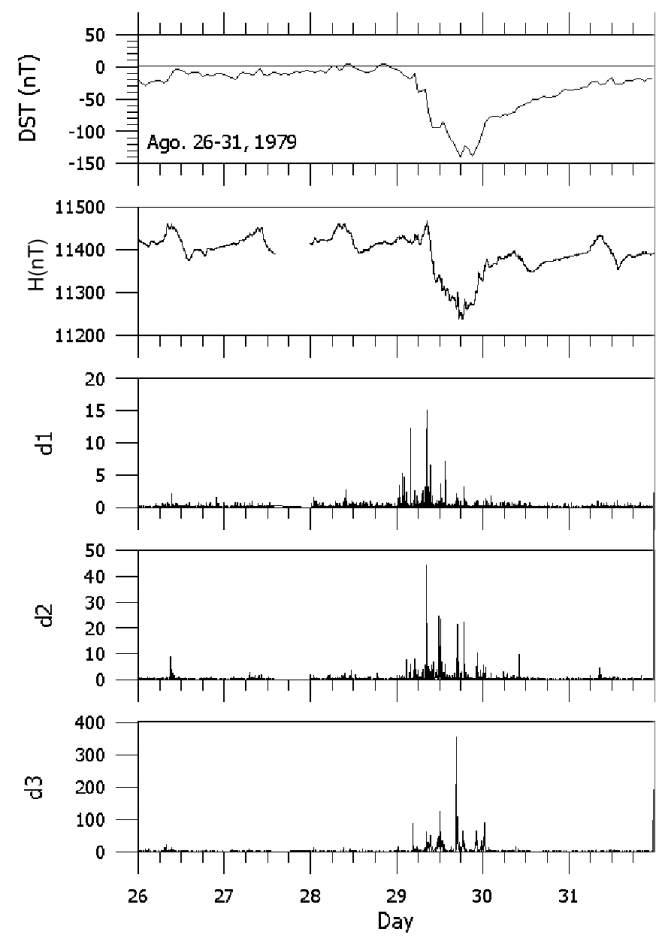


(e) DRV

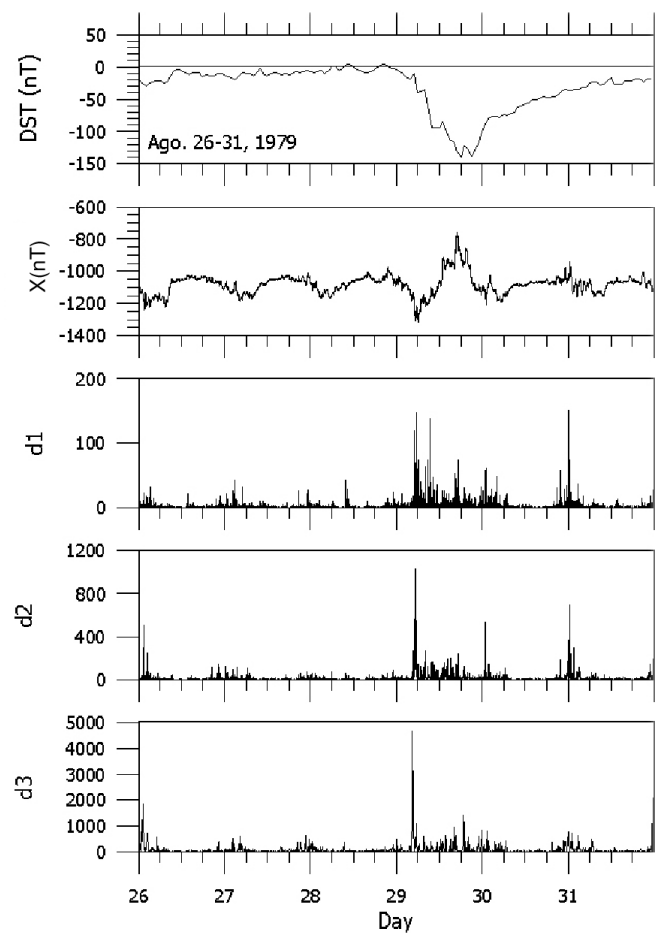

Fig. 4. Geomagnetic field data set for August, $26-31,1978$. The letters (a) to (e) stand for stations Fort Churchill, Boulder, Kakioka, Hermanus and Dumont d'Urville. Each panel shows from top to bottom, the Dst index, the $\mathrm{X}$ or $\mathrm{H}$-component of the geomagnetic field and the first three levels of the wavelet coefficients of the discrete wavelet transform. 